\title{
IROme, a New High-Throughput Molecular Tool for the Diagnosis of Inherited Retinal Dystrophies
}

\author{
Daniel F. Schorderet, ${ }^{1,2,3}$ Alexandra Iouranova, ${ }^{3}$ Tatiana Favez, \\ Leila Tiab, ${ }^{1}$ and Pascal Escher, ${ }^{1,2}$ \\ ${ }^{1}$ Institute for Research in Ophthalmology (IRO), Grand-Champsec 64, 1950 Sion, Switzerland \\ ${ }^{2}$ Department of Ophthalmology, University of Lausanne, 1004 Lausanne, Switzerland \\ ${ }^{3}$ Ecole Polytechnique Fédérale (EPFL), 1015 Lausanne, Switzerland
}

Correspondence should be addressed to Daniel F. Schorderet; daniel.schorderet@irovision.ch

Received 4 November 2012; Accepted 27 November 2012

Academic Editor: Gokce A. Toruner

Copyright (C) 2013 Daniel F. Schorderet et al. This is an open access article distributed under the Creative Commons Attribution License, which permits unrestricted use, distribution, and reproduction in any medium, provided the original work is properly cited.

\begin{abstract}
The molecular diagnosis of retinal dystrophies is difficult because of the very important number of genes implicated and is rarely helped by genotype-phenotype correlations. This prompted us to develop IROme, a custom designed in solution-based targeted exon capture assay (SeqCap EZ Choice library, Roche NimbleGen) for 60 retinitis pigmentosa-linked genes and three candidate genes (942 exons). Pyrosequencing was performed on a Roche 454 GS Junior benchtop high-throughput sequencing platform. In total, 23 patients affected by retinitis pigmentosa were analyzed. Per patient, $39.6 \mathrm{Mb}$ were generated, and 1111 sequence variants were detected on average, at a median coverage of 17 -fold. After data filtering and sequence variant prioritization, disease-causing mutations were identified in ABCA4, CNGB1, GUCY2D, PROM1, PRPF8, PRPF31, PRPH2, RHO, RP2, and TULP1 for twelve patients (55\%), ten mutations having never been reported previously. Potential mutations were identified in 5 additional patients, and in only 6 patients no molecular diagnosis could be established (26\%). In conclusion, targeted exon capture and next-generation sequencing are a valuable and efficient approach to identify disease-causing sequence variants in retinal dystrophies.
\end{abstract}

\section{Introduction}

Retinitis pigmentosa (RP) (MIM number 268000) is a group of genetically highly heterogeneous-inherited retinal dystrophies [1]. Typically, night blindness starts during adolescence, and patients progressively loose the rod photoreceptor-mediated peripheral vision. At later stages, the cone photoreceptors also become affected, constricting vision over time to the most central fovea and eventually resulting in complete blindness. To date, more than fifty genes have been linked to nonsyndromic RP (RetNet; http://www.sph.uth.tmc.edu/RetNet/). Inheritance can be autosomal dominant (AD), autosomal recessive (AR) or $\mathrm{X}$-linked, and, rarely, mitochondrial or digenic [2]. Sporadic or simplex cases account for about 30\% [3].

The molecular diagnosis of RP is difficult because (i) there is no genotype/phenotype correlation in a vast majority of patients, (ii) a high intra- and interfamilial variability of clinical phenotypes is observed in patients carrying the same causative mutation, (iii) different mutations in a same disease-linked gene cause highly variable clinical phenotypes if not clinically distinct retinal degenerations, and (iv) overlapping clinical phenotypes and disease-linked genes exist with additional retinal degenerations, that is, early-onset Leber congenital amaurosis (LCA), congenital stationary night blindness (CSNB), cone-rod dystrophies (CRD), enhanced S-cone syndrome (ESCS), or syndromic RP in Bardet-Biedl and Usher syndrome [2]. However, identification of RP-linked sequence variants is important for genetic counseling and patient management.

Similar to other Mendelian disorders, mutations in RP patients were identified until recently by linkage mapping and subsequent Sanger sequencing of candidate genes [4]. For molecular diagnosis, the validated RP mutations could be 


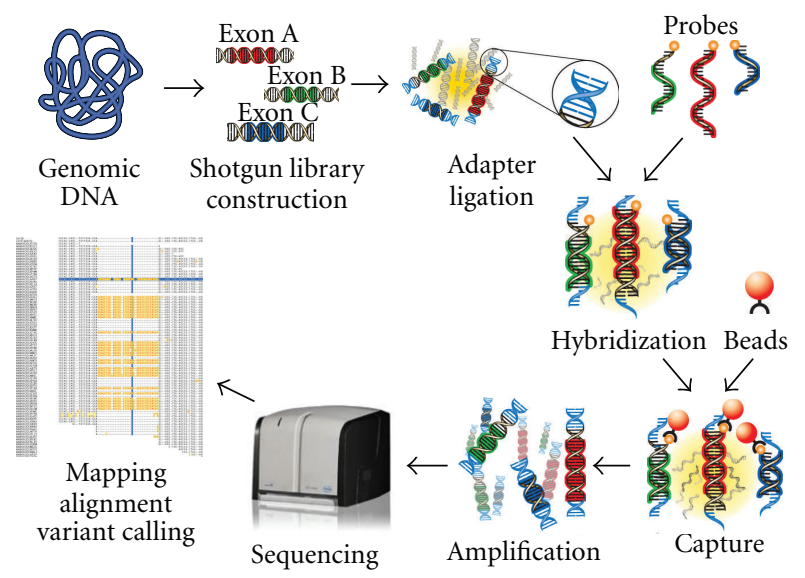

FIGURE 1: Workflow of the custom-designed targeted exome liquid hybridization capture assay IROme. Genomic DNA from patients was fragmented by nebulization and used for shotgun library construction (454 Roche GS Titanium Rapid Library). Upon adapter ligation, target enrichment is achieved by hybridizing the processed genomic DNA to biotinylated probes (Roche NimbleGen SeqCap EZ Choice). After biotin-streptavidin-based capture and washing, DNA was amplified by emulsion PCR and sequenced on a454 Roche GS Junior Sequencer. Sequencing data was aligned and mapped with the Roche 454 Reference Mapper program. Figure adapted from Roche NimbleGen technical information (http://www.nimblegen.com/products/seqcap/index.html).

detected by arrayed primer extension (APEX) chip technology [5]. However, a low success rate in detecting mutations by APEX was inherent to the genetic heterogeneity of RP patients, and in a cohort of 272 Spanish families affected by ARRP, causative mutations were identified in only $11 \%$ of them [6].

The development of next-generation sequencing (NGS) tools in recent years has allowed the production of an enormous volume of sequencing data at low costs [7]. Whole genome sequencing and downstream data handling remains cost and labor intensive, limiting its use in routine mutation detection [8]. Targeted capture of the about $30 \mathrm{Mb}$ of protein-coding regions in the human genome, the socalled exome, reduced the sequencing and data handling effort by a factor of 100 and allowed the identification of mutations in unrelated patients affected by the same syndrome [9]. Exome sequencing has since been widely used as a tool for Mendelian disease gene discovery $[10,11]$. Initially array-based, targeted sequence capture has become easy-to-use, thanks to the development of in-solution capture methods [12]. Finally, benchtop high-throughput sequencers made exome sequencing available to small-size diagnostic laboratories [13].

These technological advances prompted us to develop a custom designed in solution-based targeted capture assay, called IROme, for the detection of mutations located in the exons, including complete $3^{\prime}$-untranslated regions (UTR), intron-exon boundaries and potential promoter, and 5 '-UTR regions of 63 genes on a 454 GS Junior sequencing platform.

\section{Material and Methods}

2.1. Patients and DNA Samples. These studies were approved by the Swiss Federal Department of Health (authorization number 035.0003-48) and followed the principles of the Declaration of Helsinki. The 23 patients analyzed in this study were of Swiss, Algerian, and Tunisian origin. Blood samples were collected after informed consent. Genomic DNA was extracted from peripheral blood using a Nucleon BACC2 genomic DNA extraction kit (GE Healthcare, Glattbrugg, Switzerland). Four patients had been previously analyzed at Asper Biotech for known RP-linked mutations by APEX technology [5].

\subsection{Design of Solution-Based Capture Assay for Retinitis} Pigmentosa-Linked Genes. Exons of targeted genes were identified in the reference human genome version hg19 (http://www.ensembl.org/) (Table 1). For each exon $50 \mathrm{bp}$ were added in both $5^{\prime}$ and 3 of the exon, including the complete 3 'UTR for each gene. Potential alternative transcripts were also considered in the design. To include potential proximal promoters, an additional $1000 \mathrm{bp}$ in $5^{\prime}$ of the first exon of each gene, containing the complete 5 '-UTR, were added. The resulting custom-designed SeqCap EZ Choice library (NimbleGen, Roche) was called IROme, version 1.

2.3. GS Junior Sequencing. The workflow for GS Junior sequencing is summarized in Figure 1. DNA concentrations were measured on a NanoDrop spectrophotometer (Thermo Fisher Scientific, Wilmington, DE). 500 ng of gDNA were fragmented by nebulization, and size selected by Agencourt AMPure XP beads (Beckman-Coulter, Beverly, MA) to obtain fragments between 500 and $1200 \mathrm{bp}$. Adaptors provided in the GS Titanium Rapid Library Preparation Kit (Roche, Basel, Switzerland) were ligated to the fragmented DNA and then quantified by fluorometry (QuantiFluor, Promega, Madison, WI). This library was amplified by ligation-mediated (LM)-PCR using specific 454 primers. Then, $1 \mu \mathrm{g}$ of the PCR amplification product was dried down with COT-DNA (Roche) and 454-Hybridization Enhancing Primer in a Speedvac. The pellet was resuspended in NimbleGen's hybridization buffer and hybridized to the customdesigned SeqCap EZ Choice library (NimbleGen, Roche), called IROme v1, for $70 \mathrm{~h}$ at $47^{\circ} \mathrm{C}$ in a thermocycler. The captured DNA was bound to Streptavidin M-270 Beads (Invitrogen Dynal, Oslo, Norway) for $45 \mathrm{~min}$ at $47^{\circ} \mathrm{C}$ and, using a magnet support, washed with the 4 different NimbleGen buffers provided according to the manufacturer's instructions. The captured DNA-Beads were amplified by LM-PCR using the same specific 454 primers as before. Captured and noncaptured DNA was subjected to quantitative PCR on a Lightcycler480II (Roche, Basel, Switzerland) to measure the relative fold enrichment of the targeted sequences. Postcapture samples with an enrichment higher than 200-fold were further processed. According to the 454 GS Junior protocol (Roche), an emulsion PCR was done on 2 molecules per beads. After PCR, the beads were collected, washed, and bound to the Enrichment Beads. The enriched 
TABLE 1: List of genes enriched by targeted sequence capture (IROme).

\begin{tabular}{|c|c|c|c|c|c|}
\hline Gene & Alias & Chr & Chr location & Exons & Pathology \\
\hline$A B C A 4$ & RP19, STGD1, CORD3, and ARMD2 & 1 & 94458391-94586688 (rs) & 50 & ADRP, ARRP, ARCRD, and ARMD \\
\hline AIPL1 & LCA4 & 17 & 6327057-6338519(rs) & 6 & ARLCA, ADCRD \\
\hline BEST1 & RP50, BMD, and VMD2 & 11 & $61717293-61732987(\mathrm{fs})$ & 11 & ADRP, ARRP, and ADMD \\
\hline C2ORF71 & RP54 & 2 & $29284556-29297127(\mathrm{rs})$ & 2 & ARRP \\
\hline CA4 & RP17 & 17 & $58227302-58236902(\mathrm{fs})$ & 8 & ADRP \\
\hline CABP4 & CSNB2B & 11 & $67219877-67226699(\mathrm{fs})$ & 7 & ARLCA, ARCSNB \\
\hline CEP290 & LCA10, BBS14, and NPHP6 & 12 & $88442794-88535993(\mathrm{rs})$ & 53 & ARLCA, ARBBS \\
\hline CERKL & $\mathrm{RP} 26$ & 2 & $182401403-182545392(\mathrm{rs})$ & 14 & ARRP, ARCRD \\
\hline CLRN1 & RP61, USH3A & 3 & $150643950-150690786(\mathrm{rs})$ & 3 & ARRP \\
\hline CNGA1 & RP49 & 4 & 47937994-48018689 (rs) & 13 & ARRP \\
\hline CNGA2 & & $\mathrm{X}$ & $150906923-150913776$ (fs) & 6 & \\
\hline CNGB1 & $\mathrm{RP} 45$ & 16 & $57917847-58005020$ (rs) & 33 & ARRP \\
\hline$C R B 1$ & LCA8, RP12 & 1 & $197170592-197447585(\mathrm{fs})$ & 12 & ARRP, ARLCA \\
\hline$C R X$ & LCA7, CORD2 & 19 & $48325097-48364769$ (fs) & 4 & ADRP, ADLCA, ARLCA, and ADCRD \\
\hline DHDDS & RP59 & 1 & $26758773-26797785$ (fs) & 9 & ARRP \\
\hline EYS & RP25 & 6 & 64429876-66417118 (rs) & 43 & ARRP \\
\hline FAM161A & RP28 & 2 & 62051989-62081278 (rs) & 6 & ARRP \\
\hline FSCN2 & RP30 & 17 & $79495422-79504156$ (fs) & 5 & ADRP, ADMD \\
\hline GUCA1B & RP48, GCAP2 & 6 & $42152139-42162694(\mathrm{rs})$ & 4 & ADRP, ADMD \\
\hline GUCY2D & LCA1, CORD6 & 17 & 7905988-7923658 (fs) & 20 & ARLCA, ADCRD \\
\hline$I M P D H 1$ & LCA11, RP10 & 7 & $128032331-128050306$ (rs) & 17 & ADRP, ADLCA \\
\hline IMPG2 & RP56, sparcan & 3 & $100945570-101039404$ (rs) & 20 & ARRP \\
\hline IQCB1 & NPHP5 & 3 & $121488610-121553926$ (rs) & 15 & ARLCA \\
\hline KLHL7 & RP42 & 7 & $23145353-23215040$ (fs) & 12 & ADRP \\
\hline LCA5 & Lebercilin & 6 & $80194708-80247175$ (rs) & 8 & ARLCA \\
\hline LPCAT1 & AYTL2 & 5 & 1456595-1524092 (rs) & 14 & ARLCA \\
\hline LRAT & LCA14 & 4 & $155548097-155674270(\mathrm{fs})$ & 4 & ARRP, ARLCA \\
\hline MERTK & RP38 & 2 & $112656056-112787138(\mathrm{fs})$ & 19 & ARRP \\
\hline$N R 2 E 3$ & RP37, PNR & 15 & $72084977-72110559$ (fs) & 8 & ADRP, ARRP, and ARESCS \\
\hline$N R L$ & RP27 & 14 & $24549316-24584223(\mathrm{rs})$ & 3 & ADRP, ARRP, and ARESCS \\
\hline OFD1 & RP23 & $\mathrm{X}$ & $13752832-13787480(\mathrm{fs})$ & 23 & $\mathrm{XRP}$ \\
\hline OTX2 & & 14 & $57267426-57277197(\mathrm{rs})$ & 5 & ADLCA \\
\hline PDE6A & RP43 & 5 & $149237519-149324356$ (rs) & 22 & ARRP \\
\hline PDE6B & RP40, CSNBAD2 & 4 & 619373-664571 (fs) & 23 & ARRP, ADCSNB \\
\hline PDE6G & RP57 & 17 & $79617489-79623607$ (rs) & 4 & ARRP \\
\hline$P R C D$ & RP36 & 17 & $74523871-74541458(\mathrm{fs})$ & 5 & ARRP \\
\hline PROM1 & RP41, STGD4, CORD12, and MCDR2 & 4 & $15964699-16086001(\mathrm{rs})$ & 28 & ARRP, ADCRD, and ADMD \\
\hline$P R P F 3$ & RP18 & 1 & $150293925-150325671$ (fs) & 16 & ADRP \\
\hline PRPF6 & RP60 & 20 & $62612488-62664453(\mathrm{fs})$ & 21 & ADRP \\
\hline PRPF8 & RP13 & 17 & $1553923-1588154(\mathrm{rs})$ & 43 & ADRP \\
\hline PRPF31 & RP11 & 19 & $54618837-54635140(\mathrm{fs})$ & 14 & ADRP \\
\hline PRPH2 & RDS, RP7 & 6 & $42664340-42690312(\mathrm{rs})$ & 3 & ADRP, ADMD, ADCRD, and digenic \\
\hline$R B P 3$ & IRBP & 10 & $48381487-48390991(\mathrm{rs})$ & 4 & ARRP \\
\hline RDH12 & LCA13, RP53 & 14 & $68168603-68201169$ (fs) & 8 & ADRP, ARLCA \\
\hline$R G R$ & RP44 & 10 & $86004809-86019716(\mathrm{fs})$ & 7 & ADRP, ARRP, and ADCA \\
\hline $\mathrm{RHO}$ & RP4, CSNBAD1 & 3 & $129247483-129254012(\mathrm{fs})$ & 5 & ADRP, ARRP, and ADCSNB \\
\hline$R L B P 1$ & CRALBP & 15 & 89753098-89764922 (rs) & 9 & ARRP \\
\hline ROM1 & & 11 & $62379194-62382592(\mathrm{fs})$ & 3 & ADRP, digenic \\
\hline$R P 1$ & & 8 & $55471729-55682531(\mathrm{fs})$ & 4 & ADRP, ARRP \\
\hline$R P 2$ & & $\mathrm{X}$ & $46696375-46741793$ (fs) & 5 & XRP \\
\hline$R P 9$ & PAP1 & 7 & $33134409-33149013$ (rs) & 7 & ADRP \\
\hline
\end{tabular}


TABLE 1: Continued.

\begin{tabular}{lccccc}
\hline Gene & Alias & Chr & Chr location & Exons & Pathology \\
\hline RPE65 & LCA2, RP20 & 1 & $68894505-68915642(\mathrm{rs})$ & 14 & ARRP, ARLCA \\
RPGR & RP3, CORDX1 & $\mathrm{X}$ & $38128424-38186817(\mathrm{rs})$ & 19 & XRP, XCRD, XMD \\
RPGRIP1 & LCA6, CORD13 & 14 & $21756098-21819460(\mathrm{fs})$ & 24 & ARLCA, ARCRD \\
SAG & RP47, Arrestin & 2 & $234216309-234255701(\mathrm{fs})$ & 16 & ARRP, ARCSNB \\
SEMA4A & RP35, CORD10 & 1 & $156117157-156147543(\mathrm{fs})$ & 16 & ADRP, ARRP, and ADCRD \\
SNRNP200 & RP33 & 2 & $96940074-96971297(\mathrm{rs})$ & 45 & ADRP \\
SPATA7 & LCA3 & 14 & $88851268-88936694(\mathrm{fs})$ & 12 & ARLCA \\
TOPORS & RP31 & 9 & $32540542-32552551(\mathrm{rs})$ & 3 & ADRP \\
TUB & & 11 & $8040791-8127659(\mathrm{fs})$ & 13 & ARRP, ARLCA \\
TULP1 & LCA15, RP14 & 6 & $35465651-35480715(\mathrm{rs})$ & 15 & ARRP \\
USH2A & RP39 & 1 & $215796236-216596738(\mathrm{rs})$ & 73 & 4 \\
ZNF513 & RP58 & 2 & $27600098-27603657(\mathrm{rs})$ & ARRP \\
\hline
\end{tabular}

Genes are listed alphabetically according to their official gene symbol, and, in addition, gene aliases commonly used in ophthalmic research provided. Chromosomal (chr) location is based on the Homo sapiens high-coverage assembly GRCh37, yielding in the UCSC hg19 database (fs: forward strand; rs: reverse strand). For each gene the number of exons is listed. Targeted sequence capture was directed against genes causing autosomal dominant (AD), autosomal recessive $(\mathrm{AR}) \mathrm{X}$-linked $(\mathrm{X})$, retinitis pigmentosa $(\mathrm{RP})$, and Leber congenital amaurosis (LCA). Other retinopathies caused by a given gene are also indicated: cone or cone-rod dystrophy (CRD), macular degeneration (MD), congenital stationary night blindness (CSNB), Bardet-Biedl syndrome (BBS), enhanced S-cone syndrome (ARESCS), and chorioretinal atrophy (CA). Heterozygote ROM1 and PRPH2 mutations cause digenic disease. ORF15 of RPGR was not included in the assay.

DNA was then eluted and quantified with the provided bead counter. Sequencing was performed following the 454 GS Junior protocol. Briefly, 500'000 enriched DNA beads were mixed with Packing Beads. Then, the PicoTiterPlate (PTP) was sequentially loaded with Prelayer Beads, DNA-Packing Beads, Postlayer Beads, and PPiase Beads. Finally, the PTP was mounted in the 454 GS Junior Sequencer, and the program was run in full processing for shotgun sequencing.

2.4. Data Analysis. The workflow for data analysis and data validation is summarized in Figure 2. Sequencing data (.sff file) were analyzed with Roche 454 Reference Mapper program. Reference text (ref.txt) for gene annotations and the snp131 version of the single nucleotide polymorphism database (snp131.txt) were downloaded from the Golden Path database ( http://www.genome.ucsc.edu/). The sequence variants provided by the $454 \mathrm{HCDiffs}$.txt file were filtered for known SNPs (http://www.ensembl.org/ Homo_sapiens/Gene/Variation_Gene/), type of amino acid changes (http://genetics.bwh.harvard.edu/pph2/), and repetitive sequences. An additional in-house developed program was used to check the remaining SNPs against reference sequences obtained in Ensembl. Sequence variants were further prioritized according to inheritance, if family information was available, and to the percentage of reads containing a given sequence variant (threshold at $20 \%$ ). To analyze the coverage, scripts were written to extract global coverage data from the 454AlignmentInfo.tsv file (unique depth, column 5) and the quality of coverage at each targeted nucleotide (column 4). Part of the sequencing data was analyzed by Sequence Pilot version 3.5 (JSI Medicals, Kippenheim, Germany).

2.5. Data Validation. Sanger sequencing validated all potential pathogenic sequence variants. Briefly, 20-bp primers flanking the given region and yielding amplicons of 300-600 bp were designed (primer sequences available on request). The polymerase chain reaction (PCR) was performed in a total volume of $20 \mu \mathrm{L}$, containing $20 \mathrm{ng}$ genomic DNA, $1 \mathrm{mM}$ of each primer (Eurogentec, Liège, Belgium), and $10 \mu \mathrm{L}$ FastStart PCR Master Mix (Roche, Basel, Switzerland). Amplification was performed in a GeneAmp 9700 thermal cycler (Applied Biosystems, Carlsbad, CA, USA) with the following conditions: $1 \mathrm{~min}$ at $95^{\circ} \mathrm{C}, 35$ cycles of $1 \mathrm{~min}$ at $94^{\circ} \mathrm{C}, 1 \mathrm{~min}$ at $58^{\circ} \mathrm{C}, 1 \mathrm{~min}$ at $72^{\circ} \mathrm{C}$, and, a final elongation step at $72^{\circ} \mathrm{C}$ for $10 \mathrm{~min}$. PCR-amplified products were purified with an Invitek MSB Spin PCRapace kit (STRATEC Molecular GmbH, Berlin, Germany). Sanger sequencing was done in a final reaction volume of $10 \mu \mathrm{L}$, using BigDye Terminator v3.1 (Applied Biosystems) with forward and reverse primers. Fragments were separated on an ABI PRISM 3100 genetic analyzer (Applied Biosystems). Sequences were analyzed using Chromas 2.23 software (Technelysium, Tewantin, QLD, Australia).

\section{Results and Discussion}

3.1. IROme: Design and Validation of the Assay. The vast genetic heterogeneity of RP prompted us to develop a custom-designed hybridization-based targeted exon capture assay, called IROme. Enrichment was targeted towards a total of 63 genes (942 exons), of which 60 genes were linked to RP, LCA, and related retinal dystrophies (Table 1). The exon ORF15 of RPGR was not included in the assay because of the presence of repetitive sequences. Two RP- or LCAlinked genes, $I D H 3 B$ and $R D 3$, had been reported only in a single family so far and were not included in this version of IROme. Conversely, two candidate genes that were linked to retinal degeneration in mice, but not humans, were added to the assay (TUB and LPCAT1). A third candidate gene 


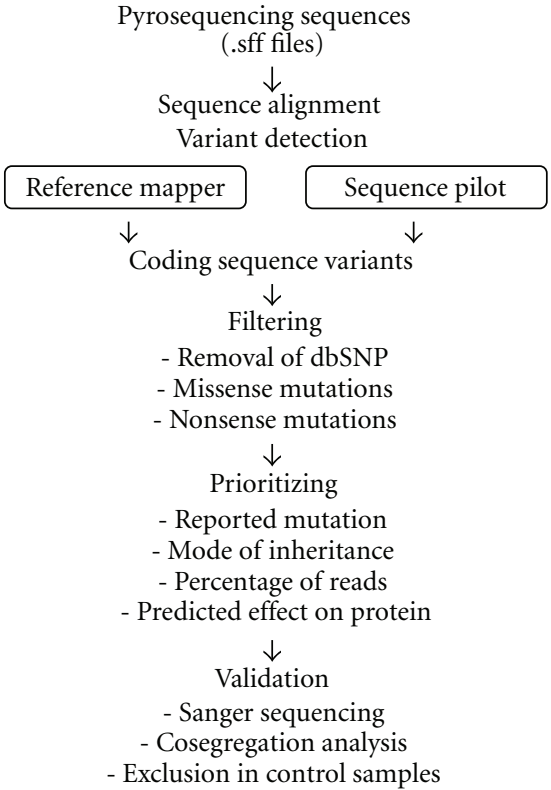

FIGURE 2: Workflow of data analysis and filtering. The sff (sequence file format) files generated by 454 Roche GS Junior sequencing were imported either into Reference Mapper or Sequence Pilot software. The coding sequence variants were selected from the 454_HCDiffs.txt files that contained all sequence variants. During filtering, coding sequence variants reported in dbSNP were removed, and missense and nonsense mutations kept. The remaining coding sequence variants were prioritized according to known reported mutations, the mode of inheritance, the percentage of sequence reads reporting the variant (threshold of $20 \%$ ), and the predicted effect on the protein (PolyPhen score).

located on chromosome X, CNGA2, was included because of its homology to CNGA1. The total of targeted regions spans $394^{\prime} 758$ bp.

Of note, after the design of IROme was completed, TTC8 (BBS8/RP51), C8ORF37, and MAK were linked to RP, and KCNJ13 and NMNAT1 to LCA. These latter genes, as well as $I D H 3 B$ and $R D 3$, will be included in a future version of IROme.

Patients 1-4 had previously been investigated by APEX technology for known RP-linked mutations [5]. All nucleotides tested by APEX were correctly detected by IROme, with a $98.9 \%$ accuracy of the sequence reads for nucleotides at a homozygous state (Table 2). A p.USH2AV2562A mutation had been detected by APEX in patient 2 in a heterozygous state, and this was correctly validated by IROme ( $46.8 \%$ of the sequence reads at 47 -fold coverage).

As an additional control, the IROme assay was tested on genomic DNA of a previously described family of Algerian origin, affected by LCA or early onset retinal degeneration [14]. The causative 6-base in-frame duplication c. TULP11593_1598dupTTCGCC was readily detected in exon 15 (Table 3, patient 5).

3.2. IROme: Variant Detection, Coverage, and Data Filtering. A total of $23 \mathrm{RP}$ patients were analyzed by IROme (Table
TABLE 2: Validation of IROme by APEX.

\begin{tabular}{lcccc}
\hline $\begin{array}{l}\text { Pat } \\
\text { number }\end{array}$ & $\begin{array}{c}\text { nt tested } \\
\text { by APEX }\end{array}$ & $\begin{array}{c}\text { nt detected } \\
\text { by IROme }\end{array}$ & $\begin{array}{c}\text { Mean } \\
\text { cvg }\end{array}$ & $\begin{array}{c}\text { \% reads } \\
\text { homo }\end{array}$ \\
\hline 1 & 557 & $100 \%$ & 25 & 98.9 \\
2 & 558 & $100 \%$ & 26 & 99.4 \\
3 & 558 & $100 \%$ & 22 & 99.2 \\
4 & 547 & $100 \%$ & 20 & 98.3 \\
\hline
\end{tabular}

The nucleotides (nt) tested by APEX represent validated RP-linked mutations or variants. The mean coverage ( $\mathrm{cvg}$ ) refers to the average of the coverage of all exons where the mutations are located. The percentage of sequence reads generated by IROme and correctly calling the nucleotides at homozygous state are indicated.

3). Pyrosequencing generated an average of $39.6 \pm 14.1 \mathrm{Mb}$ per patient, with an average read length of $408 \pm 48 \mathrm{bp}$. These long read lengths are comparable to published analyses, where the Roche 454 GS Junior generated the longest read lengths, in comparison to the other benchtop highthroughput sequencing platforms, MiSeq (Illumina) and Ion Torrent PGM (Life Technologies) [13].

On average per patient, $1111.7 \pm 222.2$ sequence variants were found (range: $736-1$ ' 826 ). Among these, $90.1 \pm 10.0$ were located in coding sequences, and a further $42.1 \pm 4.7$ were changing the amino acid sequence. By considering all patients, the median coverage was 17 -fold, with a maximal 112 -fold coverage in one exon of patient 16 (Figure 3 ). No coverage was observed for four exons (0.3\%): exons 1 of RP9, IMPDH1, and LPCAT1 and an alternative exon 2 of CNGA2. These exons contained GC-rich and/or repetitive sequences impeding efficient probe design and targeting [15]. Another 15 exons were not covered in all patients (1.6\%). Because these exons were not restricted to the 5 regions, absence of coverage was attributed to technical limitations or, as observed for patient 9, to a deletion (see below).

For patients 20 and 21, two potential heterozygote mutations had been detected at $22.6 \%$ (53-fold coverage) and $21.3 \%$ (61-fold coverage), respectively. However, these two sequence variants could not be validated by Sanger sequencing. For further patient analyses, a more stringent threshold up to $35 \%$ of sequence reads might be used for prioritization of sequence variants. Alternatively, a dynamic threshold could be implemented, starting at a high stringency and going down until one or two mutations are identified.

In conclusion, the design of IROme resulted in an over 98\% coverage of the targeted exons. The variant detection workflow could be improved by further increasing the quality of the sequencing data, that is, by using a benchtop sequencer less prone to homopolymer-associated insertion/deletion errors (e.g., MiSeq, Illumina) [13] and high-fidelity DNA polymerases [16].

3.3. IROme: Molecular Diagnosis on RP Patients. IROme analysis yielded in definite diagnosis for $55 \%$ of the RP patients, that is, 12 out of 23 patients (Patients 4, 5, 8, $9,10,11,12,13,16,17,19$, and 23). This was in line with the approximately $60 \%$ success rate reported for exome 


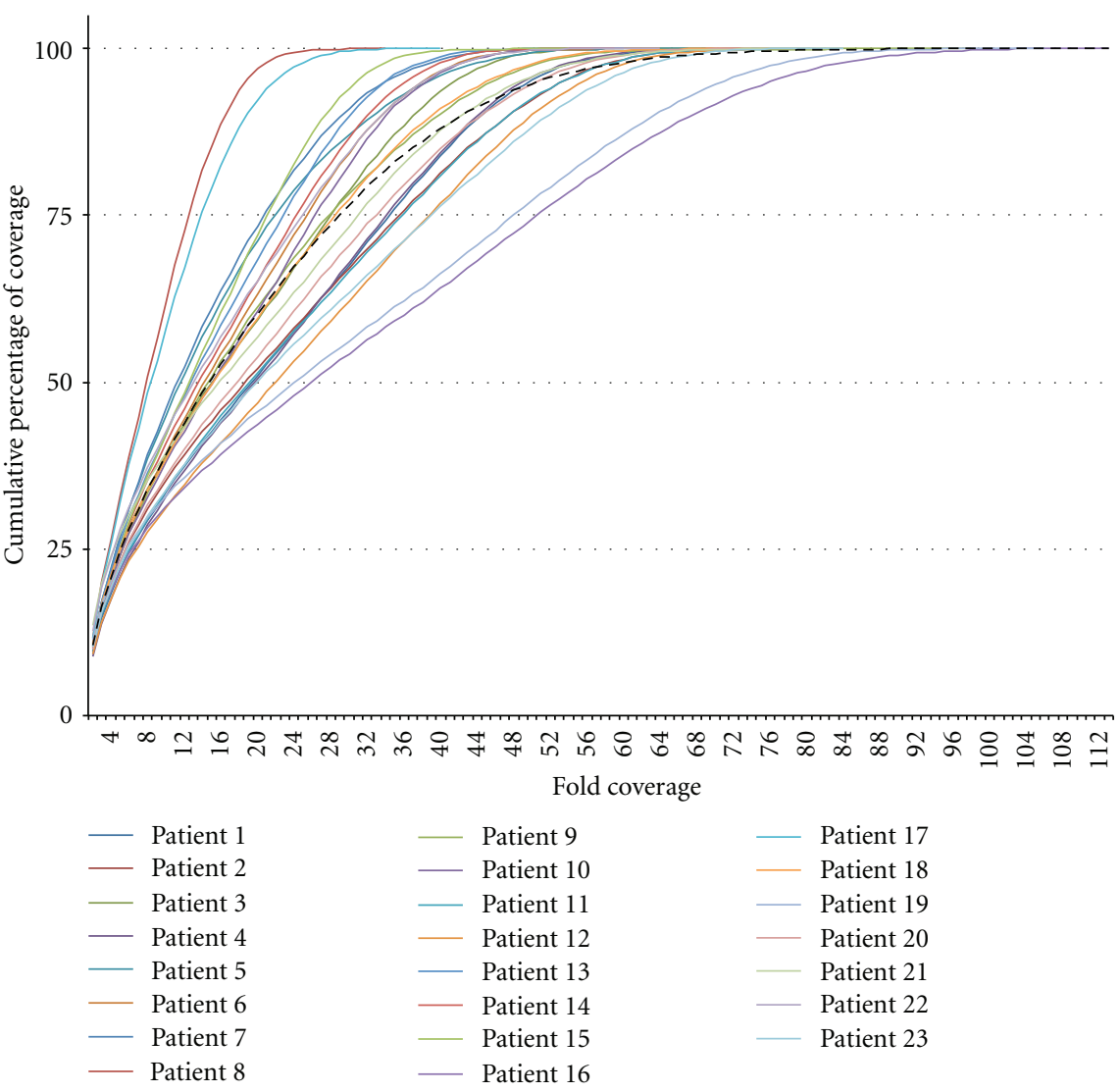

FIGURE 3: Fold coverage of targeted sequences. For each patient the unique depth data provided by column 5 of the 454 AlignmentInfo.tsv file was used to estimate the coverage per targeted bp. The onefold coverage data corresponding to reference genome sequences used for alignment purposes, but not targeted by IROme, were removed. The coverage data is represented as cumulative percentage; that is, indicating what percentage of targeted bp has a minimal coverage of $x$-fold ( $x$ axis represents the fold coverage). The average coverage for all patients is represented as a black dashed line, and the median coverage for all patients is 17 -fold.

capture strategies to identify Mendelian disease genes [4], but represented a 5 -fold increase in mutation detection as compared to the APEX assay [6]. A solution-based targeted exon capture assay similar to IROme had also identified disease-causing mutations in 11 out of 17 families affected by various retinal degenerations (65\%) [17]. In contrast, in a cohort of $100 \mathrm{RP}$ patients, array-based targeted exon capture resulted in the identification of pathogenic mutations in 36 individuals (36\%) [15]. Amplicon-based approaches identified potential mutations in $24 \%$ of patients affected by retinal degenerations (5/21) [18], in 79\% of ADRP patients $(15 / 19)$ [19], and 24\% of LCA patients (4/17) [20].

In addition to the control (patient 5), only the p.PROM1R373C mutation identified in patient 10 had been previously described [21], further underscoring the importance of screening RP-linked genes for the presence of new mutations.

The workflow for variant detection was not immediately successful for two patients. For patient 9, a deletion of exons 45-47 in ABCA4 was only found by analyzing the coverage data. For patient 16 , the $33 \mathrm{bp}$ insertion in PRPF31 was detected by Sequence Pilot, but not Reference Mapper software.
Potential mutations were found in three patients (13\%). Patient 1 inherited from her healthy mother a heterozygous p.C2ORF71-R571delRTVVPP mutation and from her healthy father a heterozygous p.FSCN2-P231S mutation. Digenic RP has been linked so far to heterozygous PRPH2 and ROM1 mutations [2], and further analyses will be necessary to validate this molecular diagnostic. Patient 2 and 20 had, respectively, two and one potential mutation, but no family members were available to confirm the result.

Results were questionable for two additional patients. Patient 6 carried a p.RHO-R252P mutation that had been previously reported [22]. However, unaffected family members were not available to confirm this dominant mutation. Also, a heterozygous p.CRX-Q105X sequence variant was detected in patient 14, but his healthy mother was also carrying it.

Finally, no molecular diagnostic could be established for six patients (26\%): in patients 18 and 21 no potential mutations were found by IROme analysis, in patients 7 and 15 the potential mutation did not segregate with disease in the family, and in patients 3 and 22 heterozygous mutations were found in genes only reported for recessive inheritance (CLRN1, EYS). 
TABLE 3: Synopsis of molecular diagnostic on RP patients by IROme.

\begin{tabular}{|c|c|c|c|c|c|c|c|c|c|c|c|c|}
\hline $\begin{array}{l}\text { Pat } \\
\text { number }\end{array}$ & $\begin{array}{c}\text { Total } \\
\text { seq } \\
\mathrm{Mb}\end{array}$ & $\begin{array}{c}\text { Read } \\
\text { length } \\
\text { bp }\end{array}$ & $\begin{array}{l}\text { Median } \\
\text { fold } \\
\text { cvg }\end{array}$ & $\begin{array}{l}\text { Total } \\
\text { seq } \\
\text { var }\end{array}$ & $\begin{array}{l}\text { cds } \\
\text { seq } \\
\text { var }\end{array}$ & $\begin{array}{l}\text { filt. } \\
\text { seq } \\
\text { var }\end{array}$ & $\begin{array}{l}\text { prio. } \\
\text { seq } \\
\text { var }\end{array}$ & $\begin{array}{c}\text { Test/val } \\
\text { seq } \\
\text { var }\end{array}$ & $\begin{array}{l}\text { Potential } \\
\text { mutation }\end{array}$ & $\begin{array}{l}\text { cvg } \\
\text { pot } \\
\text { mut }\end{array}$ & $\begin{array}{l}\text { mut } \\
\text { reads } \\
\%\end{array}$ & $\begin{array}{l}\text { Cosegregate } \\
\text { family }\end{array}$ \\
\hline 1 & 47 & 453 & 21.3 & 1206 & 114 & 51 & 8 & $2 / 2$ & $\begin{array}{c}\text { p.C2ORF71- } \\
\text { R571_P576del } \\
\text { p.FSCN2-P231S }\end{array}$ & $\begin{array}{l}38 \\
25\end{array}$ & $\begin{array}{c}55.3 \\
44\end{array}$ & $\begin{array}{l}\text { M het norm } \\
\text { F het norm }\end{array}$ \\
\hline 2 & 47.6 & 433 & 20.9 & 1217 & 98 & 44 & 7 & $2 / 2$ & $\begin{array}{l}\text { p.PDE6B-H337R } \\
\text { p.OTX2-G222R }\end{array}$ & $\begin{array}{l}21 \\
52\end{array}$ & $\begin{array}{c}100 \\
48\end{array}$ & $\begin{array}{l}? \\
?\end{array}$ \\
\hline 3 & 42.2 & 416 & 17.0 & 1085 & 78 & 39 & 6 & $1 / 1$ & p.CLRN1-P134L & 19 & 68.4 & $?$ \\
\hline 4 & 44.6 & 395 & 21.6 & 1173 & 95 & 42 & 5 & $3 / 3$ & p.RHO-Y191C & 39 & 38.5 & yes \\
\hline 5 & 24.4 & 429 & 13.8 & 894 & 104 & 45 & 1 & $1 / 1$ & $\begin{array}{l}\text { p.TULP1- } \\
\text { F529_A530dup }\end{array}$ & 6 & 100 & yes \\
\hline 6 & 31.7 & 422 & 16.2 & 1039 & 85 & 47 & 1 & $1 / 1$ & p.RHO-R252P & 22 & 54.5 & $?$ \\
\hline 7 & 20.1 & 281 & 13.3 & 789 & 77 & 38 & 4 & $2 / 2$ & $\begin{array}{c}\text { p.SAG-E11K } \\
\text { p.IMPG2-G684R }\end{array}$ & $\begin{array}{l}30 \\
34\end{array}$ & $\begin{array}{l}56.7 \\
38.2\end{array}$ & $\begin{array}{l}\text { no } \\
\text { no }\end{array}$ \\
\hline 8 & 13.9 & 445 & 9.1 & 736 & 70 & 33 & 2 & $1 / 1$ & p.RP2-D161Y & 22 & 45.5 & yes \\
\hline 9 & 29 & 297 & 17.3 & 832 & 80 & 39 & 9 & $1 / 1$ & g.ABCA4-ex45-47del & 0 & 0 & yes \\
\hline 10 & 37.3 & 443 & 16.7 & 1247 & 93 & 46 & 3 & $3 / 3$ & p.PROM1-R373C & 32 & 50 & yes \\
\hline 11 & 50.2 & 440 & 21.5 & 1151 & 92 & 46 & 2 & $1 / 1$ & p.RP2-E20X & 28 & 67.8 & yes \\
\hline 12 & 49.1 & 394 & 23.8 & 1116 & 94 & 39 & 9 & $4 / 4$ & p.CNGB1-R765C & 30 & 100 & yes \\
\hline 13 & 33 & 436 & 14.6 & 1017 & 85 & 33 & 3 & $2 / 2$ & p.GUCY2D-V887G & 18 & 94.4 & yes \\
\hline 14 & 32.6 & 443 & 15.3 & 1205 & 93 & 42 & 3 & $1 / 1$ & p.CRX-Q105X & 17 & 58.8 & M het norm \\
\hline 15 & 32.7 & 442 & 14.2 & 1026 & 86 & 40 & 3 & $1 / 1$ & p.USH2A-P2630R & 25 & 40 & no \\
\hline 16 & 69.4 & 434 & 28.4 & 1246 & 87 & 41 & 1 & $1 / 1$ & $\begin{array}{c}\text { p.PRPF31- } \\
\text { E183_ins33bp }\end{array}$ & 74 & 40 & yes \\
\hline 17 & 16.7 & 452 & 9.8 & 861 & 82 & 43 & 2 & $2 / 2$ & p.PRPH2-L39P & 18 & 50 & yes \\
\hline 18 & 39.6 & 429 & 17.2 & 1826 & 85 & 35 & 3 & $1 / 1$ & & & & \\
\hline 19 & 66.5 & 449 & 26.4 & 1298 & 103 & 45 & 5 & $2 / 2$ & $\begin{array}{c}\text { p.PRPH2- } \\
\text { S217_dup16bp }\end{array}$ & 71 & 39.4 & yes \\
\hline 20 & 47.1 & 358 & 19.8 & 1171 & 91 & 47 & 3 & $2 / 1$ & p.C2ORF71-L889P & 23 & 39.1 & $?$ \\
\hline 21 & 47 & 363 & 17.3 & 1197 & 102 & 48 & 3 & $1 / 0$ & & & & \\
\hline 22 & 36.6 & 354 & 14.7 & 1072 & 86 & 45 & 2 & $2 / 1$ & p.EYS-D2930G & 38 & 60.5 & $?$ \\
\hline 23 & 53.3 & 393 & 22.4 & 1164 & 92 & 40 & 7 & $5 / 5$ & p.PRPF8-E2331X & 38 & 44.7 & yes \\
\hline
\end{tabular}

For each patient, the total number of $\mathrm{Mb}\left(10^{6} \mathrm{bp}\right)$ sequenced on the Roche $454 \mathrm{GS}$ Junior (total seq $\mathrm{Mb}$ ) and the average read length (read length bp) are indicated. The median fold coverage ( $\mathrm{cvg}$ ) was extracted from the unique depth information. From all the sequence variants (total seq var), first only the sequence variants located in coding sequences were analyzed (cds seq var), with filtering (filt seq var) and prioritizing (prio seq var) according to Figure 2 . The sequence variants eventually tested and validated by Sanger sequencing (test/val seq var) are also indicated. For each potential mutation, the coverage (cvg pot mut) and the percentage of sequence reads reporting the potential mutation (mut reads \%) are indicated. For cosegregation analysis, "?" indicates absence of available family members and/or simplex cases. For patients 1 and 14, the mother $(\mathrm{M})$ and/or the father $(\mathrm{F})$ are healthy heterozygous carriers (het norm).

Of note, all these patients carry novel sequence variants in noncoding regions. To prioritize for potential disease-causing sequence variants in these regions, systematic annotation should not only cover splicing sites, $5^{\prime}$ - and $3^{\prime}$-UTRs, but also implement detailed information about transcription factor binding sites and regulatory elements located in the potential proximal promoter regions. Promoter sequence variants could then be tested by reporter transactivation assays (e.g., luciferase reporter assays), but this time-consuming approach cannot be implemented in a routine molecular diagnostic lab.

\section{Conclusions}

The custom designed in solution-based targeted exon capture assay IROme efficiently detected disease-causing mutations in $55 \%$ of RP patients (12/23). A $99.7 \%$ coverage of the targeted regions was obtained. The first translated exon often contains sequences with a high GC content in its $5^{\prime}$-UTR that hinders an efficient capture [23]. Remarkably, more than $95 \%$ of exons $1(60 / 63)$ were successfully enriched by IROme.In comparison, a pilot study carried out in our 
laboratory on 25 patients using whole exome sequencing (SureSelect, Agilent) resulted in no coverage of promoter regions, highly variable coverage of $3^{\prime}$-UTRs, and several genes had their first translated exon very poorly covered. For instance, the first exons of the following RP-linked genes could not be correctly analyzed: C2ORF71, CA4, CABP4, CERKL, CNGA1, FAM161A, FSCN2, GUCY2D, IMPDH1, LPCAT1, MERTK, RDH12, RP9, and RPGR (D. F. Schorderet, unpublished results). It is tempting to speculate that the additional sequences upstream of exon 1 included in IROme further enhanced the performance of the NimbleGen exome capture technology, that reportedly has more specific targeting and a higher percentage of on-target reads than competing products $[23,24]$. However, because the costs for whole exome sequencing have dramatically decreased to about 1000 \$ per patient, this method may in the future replace target enrichment and resequencing, providing that a new line of "whole exome" kits covering effectively all exons, including the first one, of all genes, will become commercially available [24].

Meanwhile, custom-designed target enrichment and subsequent next-generation sequencing are a cost-efficient approach for the molecular diagnosis of retinal dystrophies, also with respect to the relative ease of data handling and analysis [25]. Finally, the median global coverage of 17fold observed with the IROme assay also indicated the possibility to include additional retinal degeneration-linked genes, newly discovered ones or candidate genes.

\section{Acknowledgments}

The authors thank Etienne Bagnoud for technical support in informatics. This paper is supported by Swiss National Science Foundation Grants 31003A-122269 (to P. Escher and D. F. Schorderet) and 31003A_138492 (to P. Escher).

\section{References}

[1] D. T. Hartong, E. L. Berson, and T. P. Dryja, "Retinitis pigmentosa," The Lancet, vol. 368, no. 9549, pp. 1795-1809, 2006.

[2] W. Berger, B. Kloeckener-Gruissem, and J. Neidhardt, "The molecular basis of human retinal and vitreoretinal diseases," Progress in Retinal and Eye Research, vol. 29, no. 5, pp. 335-375, 2010.

[3] S. Ferrari, E. Di Iorio, V. Barbaro, D. Ponzin, F. S. Sorrentino, and F. Parmeggiani, "Retinitis pigmentosa: genes and disease mechanisms," Current Genomics, vol. 12, no. 4, pp. 238-249, 2011.

[4] C. Gilissen, A. Hoischen, H. G. Brunner, and J. A. Veltman, "Disease gene identification strategies for exome sequencing," European Journal of Human Genetics, vol. 20, no. 5, pp. 490-497, 2012.

[5] J. Zernant, M. Külm, S. Dharmaraj et al., "Genotyping microarray (disease chip) for leber congenital amaurosis: detection of modifier alleles," Investigative Ophthalmology and Visual Science, vol. 46, no. 9, pp. 3052-3059, 2005.

[6] A. Ávila-Fernández, D. Cantalapiedra, E. Aller et al., "Mutation analysis of 272 Spanish families affected by autosomal recessive retinitis pigmentosa using a genotyping microarray," Molecular Vision, vol. 16, pp. 2550-2558, 2010.

[7] M. L. Metzker, "Sequencing technologies-the next generation," Nature Reviews Genetics, vol. 11, no. 1, pp. 31-46, 2010.

[8] L. G. Biesecker, "Exome sequencing makes medical genomics a reality," Nature Genetics, vol. 42, no. 1, pp. 13-14, 2010.

[9] S. B. Ng, E. H. Turner, P. D. Robertson et al., "Targeted capture and massively parallel sequencing of 12 human exomes," Nature, vol. 461, no. 7261, pp. 272-276, 2009.

[10] S. B. Ng, K. J. Buckingham, C. Lee et al., "Exome sequencing identifies the cause of a mendelian disorder," Nature Genetics, vol. 42, no. 1, pp. 30-35, 2010.

[11] M. J. Bamshad, S. B. Ng, A. W. Bigham et al., "Exome sequencing as a tool for Mendelian disease gene discovery," Nature Reviews Genetics, vol. 12, no. 11, pp. 745-755, 2011.

[12] L. Mamanova, A. J. Coffey, C. E. Scott et al., "Target-enrichment strategies for next-generation sequencing," Nature Methods, vol. 7, no. 2, pp. 111-118, 2010.

[13] N. J. Loman, R. V. Misra, T. J. Dallman et al., "Performance comparison of benchtop high-throughput sequencing platforms," Nature Biotechnology, vol. 30, no. 5, pp. 434-439, 2012.

[14] A. Mataftsi, D. F. Schorderet, L. Chachoua et al., "Novel TULP1 mutation causing leber congenital amaurosis or early onset retinal degeneration," Investigative Ophthalmology and Visual Science, vol. 48, no. 11, pp. 5160-5167, 2007.

[15] K. Neveling, R. W. Collin, C. Gilissen et al., "Next-generation genetic testing for retinitis pigmentosa," Human Mutation, vol. 33, no. 6, pp. 963-972, 2012.

[16] I. Vandenbroucke, H. Van Marck, P. Verhasselt et al., "Minor variant detection in amplicons using 454 massive parallel pyrosequencing: experiences and considerations for successful applications," Biotechniques, vol. 51, no. 3, pp. 167-177, 2011.

[17] I. Audo, K. M. Bujakowska, T. Leveillard et al., "Development and application of a next-generation-sequencing (NGS) approach to detect known and novel gene defects underlying retinal diseases," Orphanet Journal of Rare Diseases, vol. 7, no. 1, article 8, 2012.

[18] S. J. Bowne, L. S. Sullivan, D. C. Koboldt et al., "Identification of disease-causing mutations in autosomal dominant retinitis pigmentosa (adRP) using next-generation DNA sequencing," Investigative Ophthalmology and Visual Science, vol. 52, no. 1, pp. 494-503, 2011.

[19] J. Song, N. Smaoui, R. Ayyagari et al., "High-throughput retinaarray for screening 93 genes involved in inherited retinal dystrophy," Investigative Ophthalmology and Visual Science, vol. 52, no. 12, pp. 9053-9060, 2011.

[20] F. Coppieters, B. de Wilde, S. Lefever et al., "Massively parallel sequencing for early molecular diagnosis in Leber congenital amaurosis," Genetics in Medicine, vol. 14, no. 6, pp. 576-585, 2012.

[21] Z. Yang, Y. Chen, C. Lillo et al., "Mutant prominin 1 found in patients with macular degeneration disrupts photoreceptor disk morphogenesis in mice," Journal of Clinical Investigation, vol. 118, no. 8, pp. 2908-2916, 2008.

[22] E. R. Grinberg, L. I. Dzhemileva, and E. K. Khusnutdinova, "The novel R252P Mutation of the RHO gene in patients with retinitis pigmentosa from Bashkortostan," Molecular Biology, vol. 41, no. 4, pp. 746-748, 2007.

[23] P. Frommolt, A. T. Abdallah, J. Altmüller et al., "Assessing the enrichment performance in targeted resequencing experiments," Human Mutation, vol. 33, no. 4, pp. 635-641, 2012. 
[24] A. M. Sulonen, P. Ellonen, H. Almusa et al., "Comparison of solution-based exome capture methods for next generation sequencing," Genome Biology, vol. 12, no. 9, article R94, 2011.

[25] M. Fromer, J. L. Moran, K. Chambert et al., "nd statistical genotyping of copy-number variation from whole-exome sequencing depth," The American Journal of Human Genetics, vol. 91, no. 4, pp. 597-607, 2012. 

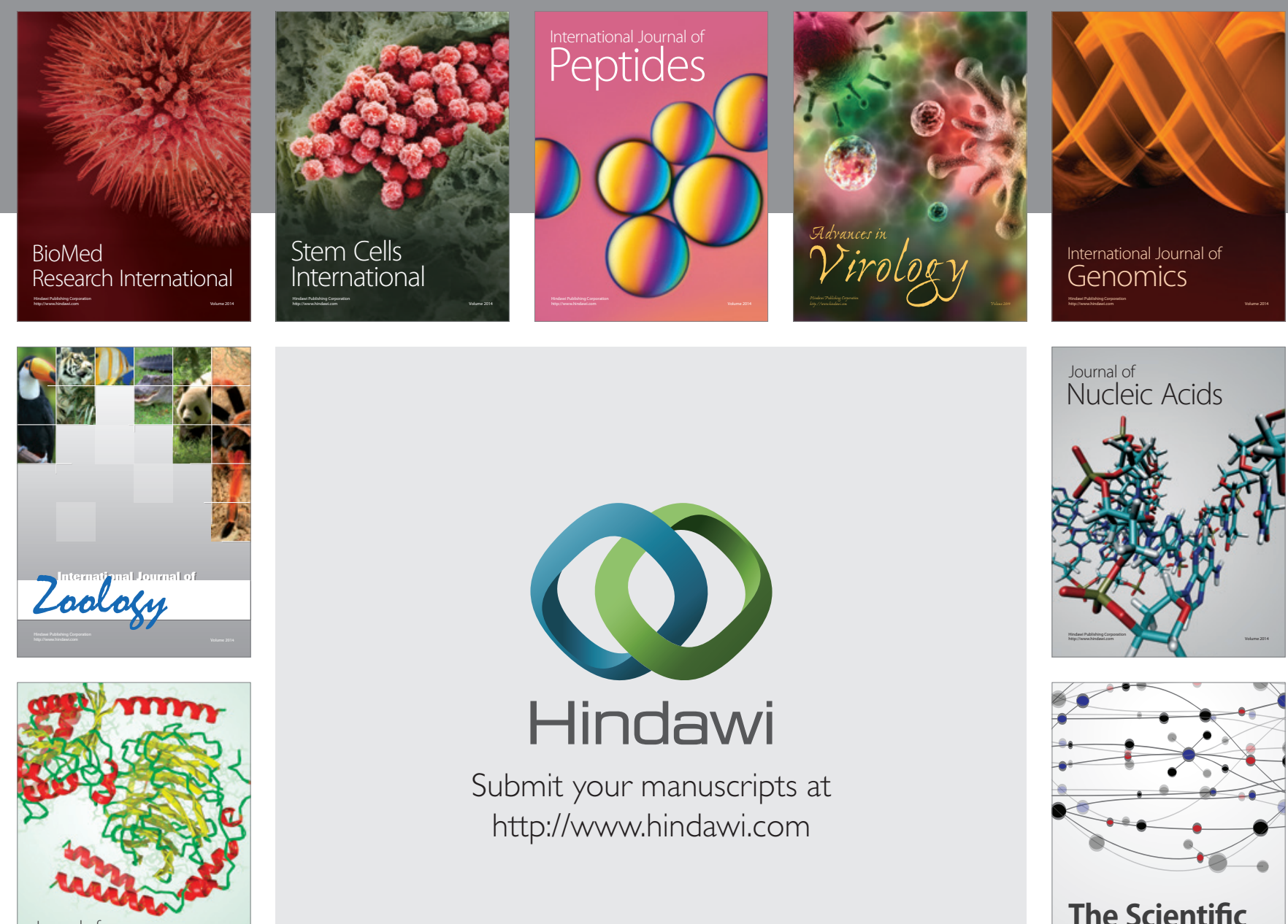

Submit your manuscripts at

http://www.hindawi.com

Journal of
Signal Transduction
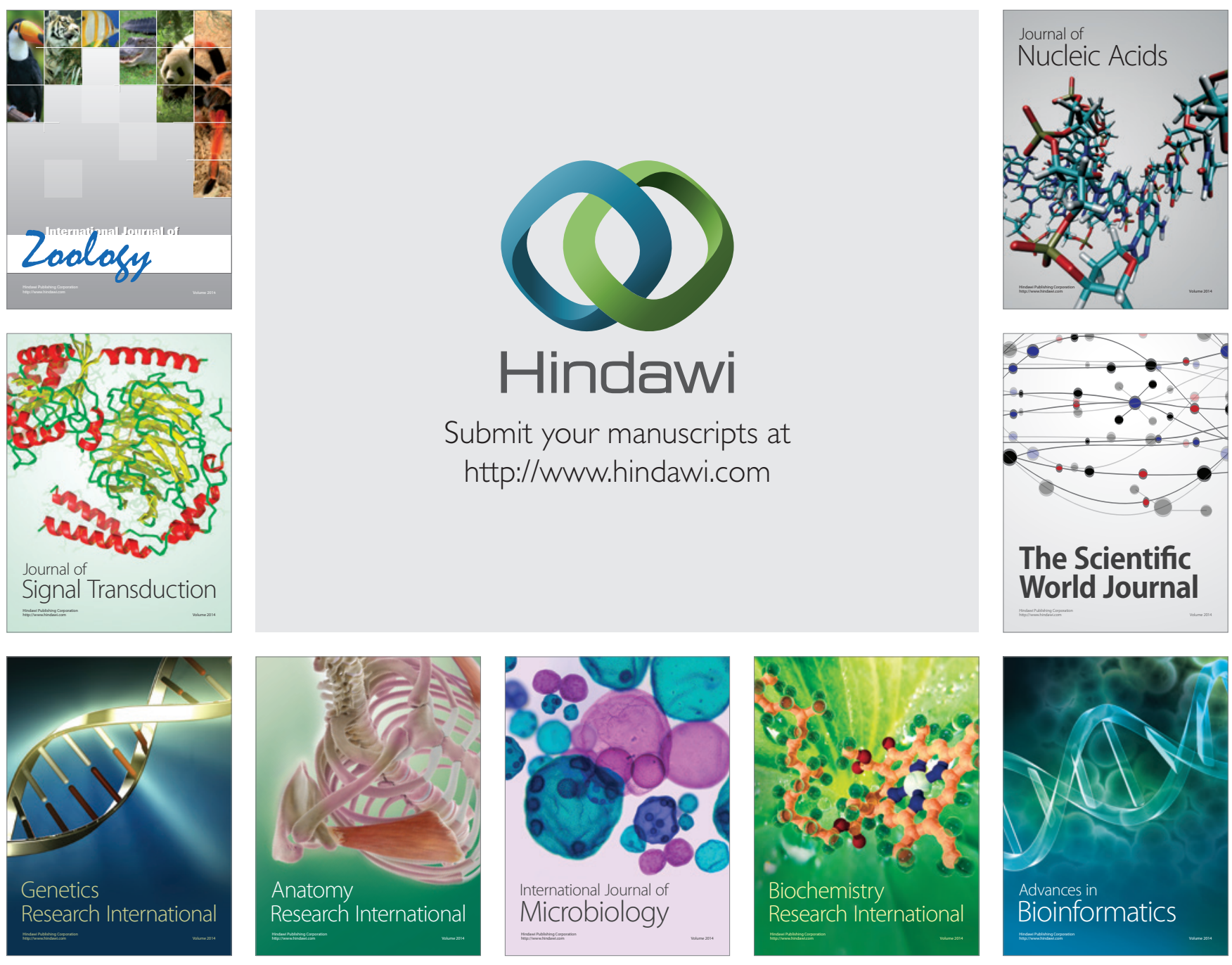

The Scientific World Journal
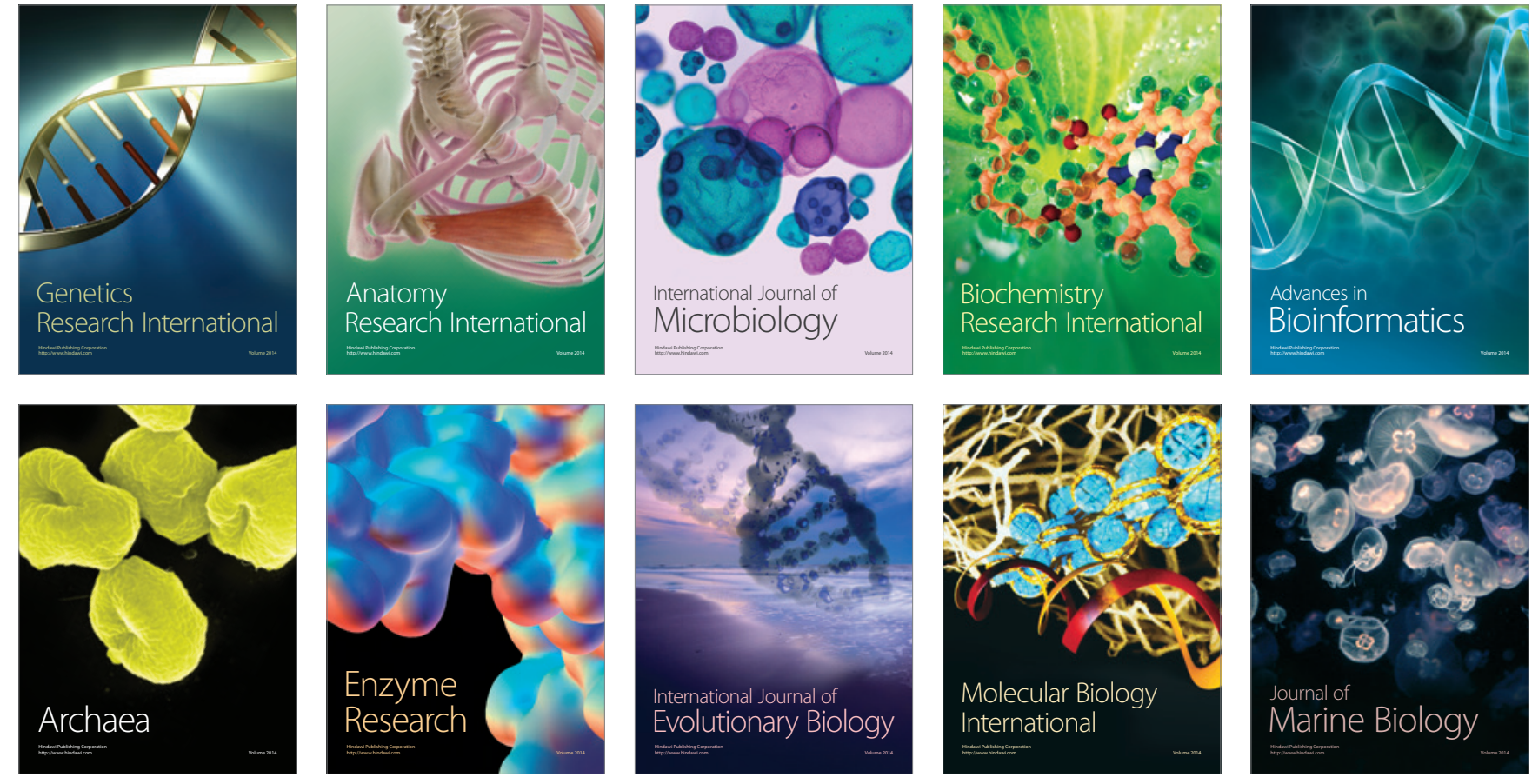